\title{
Application of Gray Cloud Model in Software Quality Evaluation
}

\author{
Shao Yong ${ }^{1, a}$, Jia Zhen ${ }^{2, b}$ and Yan Changshun ${ }^{2, c}$ \\ ${ }^{1}$ School of Software, Beijing University of Technology, Beijing 100124, China; \\ ${ }^{2}$ School of Software, Beijing University of Technology, Beijing 100124, China. \\ ashaoyong@bjut.edu.cn, b lovingtobacco@sina.com, cyuewuxing@ bjut.edu.cn
}

Keywords: software quality, gray cloud, whitening model, gray clustering.

\begin{abstract}
Based on analyzing software quality, the model provided by GB / T 16260.2 was adopted as the grounds of evaluation index system, and the index weight was determined by analytic hierarchy process (AHP). In addition, take the incompleteness and randomness of information into consideration, the evaluation model applied with Normal Cloud definite weighted function, was used to empirically evaluate and research the relevant software.
\end{abstract}

\section{Introduction}

Gray system theory has been developed rapidly and extensive applied in software engineering field in recent years[1-2], what's more, it comes into being various quantitative evaluation models of software quality, which is vital to software industry. It includes Han Xinyu's [3]software quality evaluation method based on hierarchical-gray analysis method, Liu Yuyan's [4-5]software quality evaluation model based on gray fixed weight clustering, as well as Yin Hongjian's [6]software quality gray evaluation model based on center-point triangular definite weighted function. These quantitative evaluation models of software quality are able to evaluate software quality to a certain degree. However, as for the hierarchical- gray evaluation model and gray fixed weigh clustering evaluation model are lack of adequate theoretical support, owning to they were proposed for a long time, and software quality indicators was selected only from relevant data. While as for software quality gray evaluation model based on center-point triangular definite weighted functions, it only considered the incompleteness of data information, while neglected the randomness of information, so that evaluation result is not accurate enough as it should be. So as to get over these shortcomings, this paper sets up the software quality evaluation index system in accordance to GB / T 16260.2 model, and adopts gray cloud model which has considered the incompleteness and randomness, to determine the gray class of the software and so as to evaluate the software quality.

\subsection{Gray Cloud Model Theory}

Gray cloud model was proposed by Harbin Institute of Technology doctoral student, Wang Hongli and his doctoral tutor, Professor Feng Yuqiang, and it is shown as Figure 1. In the model, $\mathrm{U}(\mathrm{x}) \in$ $[0,1]$ indicates the definite weight of the gray number, and the definite weighted model stand for a random curve with a certain uneven thickness. Suppose $U$ is a discourse domain, $U=\{x\}$, $T$ is the language value associated with $\mathrm{U}$, and the element $\mathrm{x}$ in $\mathrm{U}$ is a random number with stable tendencies compared to $\mathrm{T}$ expressed by the grey whitening weight. The distribution of whitening weight in the discourse domain is called as the albino right cloud, referred to as gray cloud.

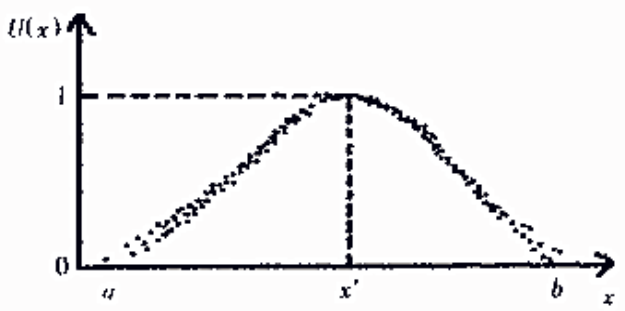

Fig. 1 Gray cloud model diagram 
The numerical characteristics of gray cloud are characterized by peak value (Cx), left and right boundary value (Lx, Rx), entropy En as well as hyper entropy He and other numerical values. The numerical characteristics of the complete gray cloud are denoted as GL (Cx; Lx, Rx; En; He). Peak value is the most representative qualitative value in gray concept, which is equal to the value of whitening right as 1 , the peak can be a number, turn into point peak; it can also be an interval, called as interval peak. If the peak is an interval, then the left end point of the interval is the left peak LCx, and the right end point is the right peak RCx. The peak, entropy and hyper entropy of the peak model are respectively:

$$
\begin{aligned}
& \mathrm{Cx}=\frac{R \mathrm{x}+L \mathrm{x}}{2} \\
& \mathrm{En}=\frac{R \mathrm{x}-L \mathrm{x}}{6} \\
& \mathrm{He}=\frac{E \mathrm{n}}{\mathrm{a}}
\end{aligned}
$$

The incompleteness and randomness of gray cloud model expression concept possess the below characteristics:

First of all, $\mathrm{x}$ to T's whitening weight is a probability distribution instead of a fixed value, so it produces a gray cloud model rather than a clear function curve.

Secondly, the gray cloud is made of a great deal of cloud droplets, one cloud droplet can be reached a realization in number of a qualitative concept expressed by a grey number. The whole gray cloud reflects the basic characteristics of the gray concept.

Thirdly, from the perspective of gray theory, the gray cloud curve is gray number's definite weighted curve.

Last but not least, the thickness of gray cloud is uneven, and its thickness reflects the randomness of the definite weighted coefficient. The more the information relative locates, the less the randomness of the definite weight is, and the converse is also true. It is similar to the reality, People are always confident with the concepts or things which they grasp more, and it is easy for them to make decisions; while as for the concepts or things which they grasp less, it's hard for them to make judgments and decisions.

The difference between the gray cloud model and the cloud model is that cloud model's ordinate stands for membership grade of fuzziness, while the gray model's ordinate stands for the definite weighted gray number. Therefore, the gray cloud model can not only represent information's incompleteness, but also represent the randomness of person's subjective judgments. It is a comprehensive model of qualitative and quantitative transformation of gray and randomness.

Normal distribution is a kind of universal distribution, in which all branches of the society and natural sciences have proved to be. The normal gray cloud model belongs to a kind of gray cloud model in line with normal distribution curve, and the mathematical expectation curve of normal gray cloud is:

$$
\operatorname{NGL}(\mathrm{x})=\exp \left[-\frac{(\mathrm{x}-C \mathrm{x})^{2}}{2\left(\frac{R \mathrm{x}-L \mathrm{x}}{6}\right)^{2}}\right]
$$

Normal gray cloud generation algorithm shows as below:

(1) In the interval [Lx, Rx], generated $\mathrm{Cx}$ as the expectation, and generated the normal random number, $\mathrm{x}_{\mathrm{i}}$, which takes $\mathrm{En}=\frac{R \mathrm{x}-L \mathrm{x}}{6}$ as the standard deviation;

(2) generate $\mathrm{En}=\frac{R \mathrm{x}-L \mathrm{x}}{6}$ as the expected value, and generate normal random number En', which takes He as the standard deviation; 
(3) calculate $\mathrm{u}_{\mathrm{i}}=\exp \left[-\frac{(\mathrm{x}-C \mathrm{x})^{2}}{2\left(E \mathrm{n}^{\prime}\right)^{2}}\right], \quad\left(\mathrm{x}_{\mathrm{i}}, \mathrm{u}_{\mathrm{i}}\right)$ as the cloud droplets.

\section{The Establishment of the Software Quality Evaluation Index System}

Software quality characteristics can be divided into six parts by the model provided by GB / T 16260.2: functionality, usability, efficiency, reliability, maintainability, and portability. These six characteristics have been generally recognized in software quality, which can reflect the nature of the software features objectively, but also easy to be understood and accepted. At the same time, the model deal with software quality in layer, go forward one by one; meanwhile, this model provides the measurement purpose, application method, measurement and formula of the lowest measurement value. It provides a scientific and objective theoretical basis for the qualitative and quantitative characterization. Beijing Software Product Quality Inspection and Testing Center has been set up on October $12^{\text {th }}, 2004$, which is an application software product quality supervision and inspection institution approved by State Administration of Quality Supervision. Beijing Software Product Quality Inspection and Testing Center, is the first national software product quality inspection and testing center among China's quality supervision system; in addition, it is also a third party software product quality testing agency which has been approved by China National Laboratory and tested by National Metrology Certification. As lots of company's data proved, the model provided by GB / T 16260.2 is effective to evaluate the software quality. Hence, this paper eventually selects the model provided by as the framework of the evaluation process, and the evaluation index system is shown in Fig.2.

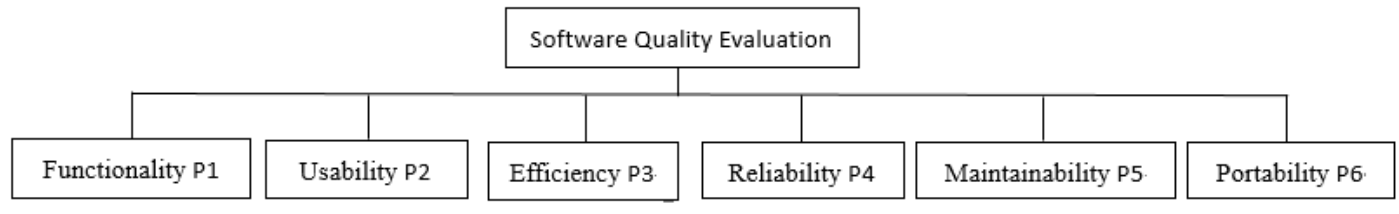

Fig. 2 Software Quality Evaluation Index System

\section{Software Quality Evaluation Based on Gray Cloud Definite Weighted Function}

The software quality evaluation of this paper, based on the grey cloud definite weighted function, divides the sample subjects into methods which can be defined categories, each category can be regarded as the collection of same class observation object. The calculation step of Normal gray cloud clustering is as follows:

Step 1 according to requiring grey class number $s$ of evaluation, select $\lambda 1, \lambda 2, \ldots, \lambda s$ as the points that are most gray $1,2, \ldots$, s. it can be the middle point of the gray value range [7], and it can also a point selected by actual situation according to the maximum probability belong to the gray, which was called as center point.

Step 2 on the basis of s grey class and center point obtained from Step 1, to establish each grey class's normal grey cloud definite weighted functions, as shown in the pictures.

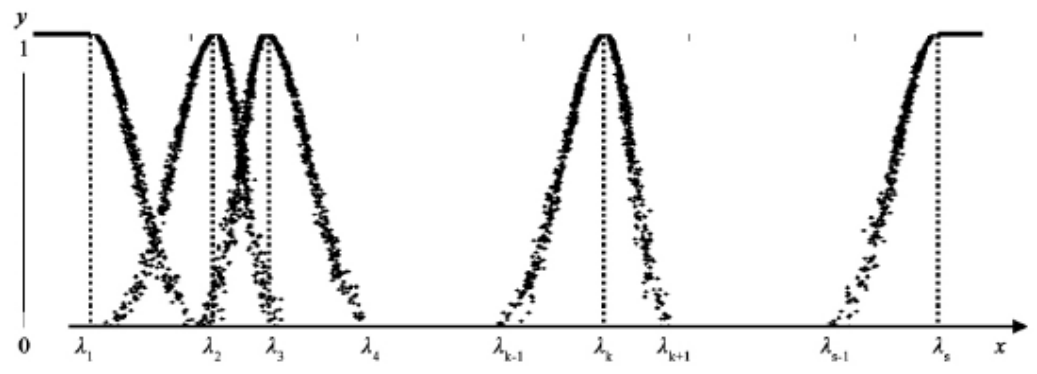

Fig. 3 Normal whitenization weight function of grey cloud

Step 3: Calculate the definite weighted value, the specific method shows as below: 
(1) Identify each indicators and the figure features of definite weighted model, then construct definite weighted model.Firstly, to provide each index's classification standard, so as to classify each index according to the evaluation of the decision makers. provide the peak value $\lambda_{\mathrm{jk}}$ of each index's grey definite weighted model, and provide the boundary value of each index definite weighted model. According to the above formula [8], calculate entropy and hyper entropy; then according to the previous normal grey cloud generated formula, generate each index's definite weighted model based on numerical characteristics of each index's definite weighted model.

(2) Calculate the clustering weight, namely to calculate the weight of index j to k grey class, that is the weight of index $\mathrm{j}$ to $\mathrm{k}$ grey class expressed as:

$\eta_{j}^{k}=\frac{\lambda_{j}^{k}}{\sum_{j=1}^{m} \lambda_{j}^{k}}$

(3) calculate the unevaluated sample object $\mathrm{i}(\mathrm{i}=1,2, \ldots, \mathrm{n})$, about the grey class $\mathrm{k}(\mathrm{k}=1,2, \ldots, \mathrm{s})$ 's integrated clustering number formula is: $\sigma_{\mathrm{i}}^{\mathrm{k}}=\sum_{\mathrm{j}=1}^{\mathrm{m}} \mathrm{f}_{\mathrm{j}}^{\mathrm{k}}\left(\mathrm{x}_{\mathrm{ij}}\right) \bullet \eta_{\mathrm{j}}^{\mathrm{k}}$, among which $f_{j}^{k}\left(x_{i j}\right)$ refers to index $j$ to k grey class’s normal grey cloud definite weighted value, $\eta_{\mathrm{j}}^{\mathrm{k}}$ refers to index j's clustering weight [9]. Thus it can be generated object i to grey class k's coefficient of grey cloud clustering:

$$
\sigma i=\left[\sigma_{i}^{1}, \sigma_{i}^{2}, \cdots \sigma_{i}^{s}\right]=\left[\sum_{j=1}^{m} f_{j}^{1}\left(x_{i j}\right) \bullet \eta_{j}^{1}, \sum_{j=1}^{m} f_{j}^{2}\left(x_{i j}\right) \bullet \eta_{j}^{2}, \cdots, \sum_{j=1}^{m} f_{j}^{s}\left(x_{i j}\right) \bullet \eta_{j}^{s}\right]
$$

(4) From the formula $\max _{1 \leq \mathrm{k} \leq \mathrm{s}}\left\{\sigma_{\mathrm{i}}^{\mathrm{k}}\right\}=\sigma_{\mathrm{i}}^{\mathrm{k} *}$, it can be conducted that object i belongs to grey class $\mathrm{k}^{*}$, namely decide the sample belongs to which level of $\mathrm{k}^{*}$, gray cloud clustering coefficient reflects the extent of its evaluation level [10].

\section{Case Analysis}

\subsection{Data collection}

According to the above model provided by GB / T 16260.2, from the perspective of software's reliability, maintainability, functionality, usability, efficiency and portability, this paper evaluates 7 software "On xx information system central platform System” combined with normal grey cloud definite weighted model and other seven software to make an assessment. Each index value of the evaluated software is shown as Table 1.

Table 1 Evaluated Software Object to Achieve The Value of The Indicators

\begin{tabular}{|c|c|c|c|c|c|c|}
\hline Index & $\begin{array}{c}\text { Functionality } \\
\text { P1 }\end{array}$ & $\begin{array}{c}\text { Usability } \\
\text { P2 }\end{array}$ & $\begin{array}{c}\text { Efficiency } \\
\text { P3 }\end{array}$ & $\begin{array}{c}\text { Reliability } \\
\text { P4 }\end{array}$ & $\begin{array}{c}\text { Maintainability } \\
\text { P5 }\end{array}$ & $\begin{array}{c}\text { Portability } \\
\text { P6 }\end{array}$ \\
\hline Software 1 & 79 & 84 & 92 & 57 & 65 & 82 \\
\hline Software 2 & 51 & 56 & 53 & 89 & 60 & 81 \\
\hline Software 3 & 64 & 85 & 86 & 93 & 96 & 73 \\
\hline Software 4 & 77 & 66 & 80 & 72 & 97 & 69 \\
\hline Software 5 & 87 & 50 & 63 & 61 & 67 & 55 \\
\hline Software 6 & 70 & 54 & 62 & 71 & 91 & 98 \\
\hline Software 7 & 83 & 59 & 88 & 94 & 90 & 75 \\
\hline
\end{tabular}

\subsection{Gray clustering calculation}

According to the requirements of evaluation, its result is divided into four categories: "excellent", "good", "medium" and "poor". Determine each index’s classification standard, namely to the peak value $\lambda \mathrm{jk}$ of each index's grey class definite weighted model, and conduct each index's definite model, provide the left and right boundary, then calculate the entropy, and determine the hyper 
entropy; then on the basis of each index's numerical features, generate each index's definite model according to normal grey cloud generated formula.

Table 2 The Grading Standards of Each Index

\begin{tabular}{|l|l|l|l|l|l|l|}
\hline & P1 & P2 & P3 & P4 & P5 & P6 \\
\hline 1 excellent & 93 & 89 & 84 & 97 & 95 & 87 \\
\hline 2 good & 81 & 70 & 71 & 90 & 80 & 79 \\
\hline 3 medium & 61 & 63 & 62 & 75 & 69 & 65 \\
\hline 4 poor & 53 & 59 & 55 & 62 & 51 & 54 \\
\hline
\end{tabular}

Taking the functional P1 as an instance, the numerical characteristics of the four levels show as below: Poor GL (53,0,60,10,1); Medium GL (61,55,75,3,0.5); Good GL (81,70,89 , 3,0.5); and Excellent GL $(93,83,100,2,0.3)$.

Fourthly, adopt the formula $\eta_{j}^{k}=\frac{\lambda_{j}^{k}}{\sum_{j=1}^{m} \lambda_{j}^{k}}(k=1,2, \ldots, s)$, to calculate the clustering weights of 4 kinds of poly objects, the result is shown as Table 3.

Table 3 Gray Clustering weights

\begin{tabular}{|c|c|c|c|c|c|c|}
\hline & P1 & P2 & P3 & P4 & P5 & P6 \\
\hline 1 & 0.1706 & 0.1633 & 0.1541 & 0.1780 & 0.1743 & 0.1596 \\
\hline 2 & 0.1720 & 0.1486 & 0.1507 & 0.1911 & 0.1699 & 0.1677 \\
\hline 3 & 0.1544 & 0.1595 & 0.1570 & 0.1899 & 0.1747 & 0.1646 \\
\hline 4 & 0.1587 & 0.1766 & 0.1647 & 0.1856 & 0.1527 & 0.1617 \\
\hline
\end{tabular}

According to normal grey cloud's definite model and the clustering class weight, recalculate the grey class's clustering coefficient, and then compute the clustering vector, and form the matrix of gray clustering.

According to the formula: $\sigma_{i}^{k}=\sum_{j=1}^{m} f_{j}^{k}\left(x_{i j}\right) \bullet \eta_{j}^{k}$ the matrix of the gray clustering vectors is shown as below:

$\left(\begin{array}{cccc}0.0640 & 0.0465 & 0.0638 & 0.0524 \\ 0.0160 & 0.0550 & 0.0175 & 0.1147 \\ 0.1363 & 0.1011 & 0.0628 & 0 \\ 0.0328 & 0.1132 & 0.1327 & 0.0371 \\ 0.0511 & 0.0446 & 0.0332 & 0.1039 \\ 0.0334 & 0.1068 & 0.100 & 0.0353 \\ 0.1196 & 0.1236 & 0.0165 & 0.0353\end{array}\right)$

Therefore, $\max _{1 \leq \mathrm{k} \leq 4}\left\{\sigma_{1}^{\mathrm{k}}\right\}=\sigma_{1}^{1}=0.0640, \max _{1 \leq \mathrm{k} \leq 4}\left\{\sigma_{2}^{\mathrm{k}}\right\}=\sigma_{2}^{4}=0.1147, \max _{1 \leq \mathrm{k} \leq 4}\left\{\sigma_{3}^{\mathrm{k}}\right\}=\sigma_{3}^{1}=0.1363$, $\max _{1 \leq \mathrm{k} \leq 4}\left\{\sigma_{4}^{\mathrm{k}}\right\}=\sigma_{4}^{3}=0.1327, \max _{1 \leq \mathrm{k} \leq 4}\left\{\sigma_{5}^{\mathrm{k}}\right\}=\sigma_{5}^{4}=0.1039, \max _{1 \leq \mathrm{k} \leq 4}\left\{\sigma_{6}^{\mathrm{k}}\right\}=\sigma_{6}^{2}=0.1068$, $\max _{1 \leq \mathrm{k} \leq 4}\left\{\sigma_{7}^{\mathrm{k}}\right\}=\sigma_{7}^{2}=0.1236$.

\subsection{Conclusion}

Calculate the above software's evaluation results through normal grey cloud model, As shown in Table 4, the quality of the software 1and 3 are excellent; the quality of software 6 and 7 are good, the software 4 is medium, the software 2 and 5 are poor. These seven software's qualities are uneven.

Table 4 Gray Cloud Evaluation Model Evaluation results

\begin{tabular}{|l|c|c|c|c|c|c|c|}
\hline Software & 1 & 2 & 3 & 4 & 5 & 6 & 7 \\
\hline Comprehensive evaluation results & Excellent & Poor & Excellent & Medium & Poor & Good & Good \\
\hline
\end{tabular}




\subsection{Contrast}

Adopting the grey cloud model with center triangular definite weighted functions, evaluate the above seven software, the result is shown as Table 5. By contrast, it can be found that quality of software 4 is medium evaluated by normal grey cloud model, but the quality of software 4 is good through adopting the grey cloud model with center triangular definite weighted functions. As the matter of fact, according to company's inspection result and user feedbacks, the quality of software 4 should be medium level. Therefore, the grey cloud model can be adopted to further improve the evaluation accuracy.

Table 5 Evaluation Results of Gray Evaluation Model for Center Point Triangle Whitening Weight Function

\begin{tabular}{|l|c|c|c|c|c|c|c|}
\hline Software & 1 & 2 & 3 & 4 & 5 & 6 & 7 \\
\hline Comprehensive evaluation results & Excellent & Poor & Excellent & Good & Poor & Good & Good \\
\hline
\end{tabular}

\section{Conclusions}

The normal gray cloud evaluation model can be adopted to determine more accurately the level of software quality, at the same time, adopting the model provided by GB / T 16260.2 can be set up a more scientific software quality evaluation index system, more responsive to software quality, which is beneficial to reflect comprehensive level of software quality. It also provides a new thought for quantitative evaluation of software quality, which has a popularization and application value in the field of software engineering.

\section{Acknowledgments}

Many people have offered me valuable help in my thesis writing, including my tutor, my classmates and my parents. Firstly, I would like to give my sincere gratitude to Prof. Shao Yong, my tutor who, with extraordinary patience and consistent encouragement, gave me great help by providing me with necessary materials, advice of great value and inspiration of new ideas. It is his suggestions that draw my attention to a number of deficiencies and make many things clearer. Without his strong support, this thesis could not been the present form. My heartfelt thanks also go to Prof. Yan Changshun, my teacher of thesis writing course, for his help in the making of this thesis as well as his enlightening lectures from which I have benefited a great deal. Then, I pleased to acknowledge my classmates for their invaluable assistance throughout the preparation of the original manuscript. They graciously make considerable comments and sound suggestions to the outline of this paper. Besides, I am extremely grateful for my roommates for their cooperation, comments, and wise advice, although their advice is not always taken, where faults and infelicities remain.

\section{References}

[1] Ke Jian Chu, Min Tan. Assessment of Sediment Quality in Jiangsu Coastal Ocean Based on Grey Clustering Method [J]. Applied Mechanics and Materials, 2014, 3044 (535): 266-271.

[2] Lukasz Radliński. A conceptual Bayesian net model for integrated software quality prediction [J]. Annales UMCS, Informatica, 2011, 11 (4): 49-60.

[3] HAN Xin-yu, TANG Long-li, LIU Bo.Research on Software Quality Evaluation Method Based on Hierarchy-gray Analysis [J]. Electronic Product Reliability \& Environmental Test, 2009,27 (3) : $1 \sim 3$

[4] LIU Yu-yan.Research on Software Quality Evaluation Model Based on Gray Fixed Weight Clustering [J]. Journal of Southwest University (Natural Science Edition)，2008，20(5) : 177 180

[5] LIU Si-feng, XIE Na-ming. A new gray evaluation method based on improved triangular whitening weight function [J]. Journal of Systems Engineering, 2011，26 
[6] YIN Hong-jian, A Gray Evaluation Model for Software Quality of Whitening Function of Center Point Triangulation [J]. Modern Computer，2010.01（3） : 45 47

[7] Elisa Valentina Oneţ. Particle Swarm Optimization and Genetic Algorithms [J]. Journal of Computer Science and Control Systems, 2009, 2 (2): 43.

[8] Huihui ,Yu,Yingyi ,Chen,Shahbaz Gul Hassan, Daoliang Li. Prediction of the temperature in a Chinese solar greenhouse based on LSSVM optimized by improved PSO [J]. Computers and Electronics in Agriculture, 2016, 122(3):94-102.

[9] Fatima ,Ardjani,Kaddour ,Sadouni. Optimization of SVM Multiclass by Particle Swarm (PSO-SVM) [J]. International Journal of Modern Education and Computer Science (IJMECS), 2010, 2 (2): 32-38.

[10] Zabir ,Haider Khan,Tasnim ,Sharmin Alin,Akter Hussain. Price Prediction of Share Market Using Artificial Neural Network 'ANN' [J]. International Journal of Computer Applications, 2011, 22 (2):42. 